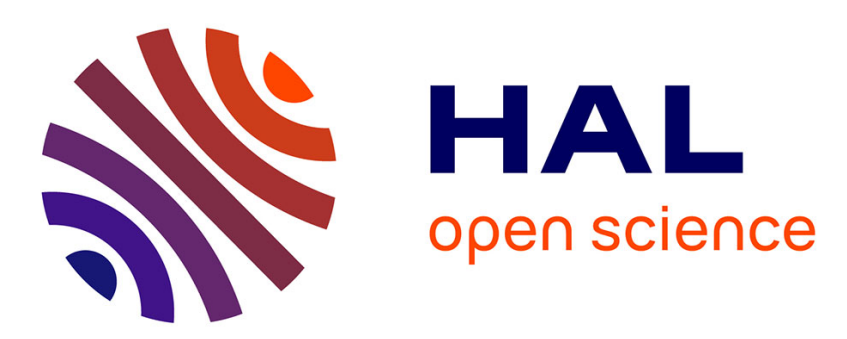

\title{
A CAD Tool for an Array of Differential Oscillators Coupled Through a Broadband Network
}

Mihaela-Izabela Ionita, David Cordeau, Jean-Marie Paillot, Smail Bachir, Mihai Iordache

\section{- To cite this version:}

Mihaela-Izabela Ionita, David Cordeau, Jean-Marie Paillot, Smail Bachir, Mihai Iordache. A CAD Tool for an Array of Differential Oscillators Coupled Through a Broadband Network. International Journal of RF and Microwave Computer-Aided Engineering, 2013, 23 (2), pp.178-187. 10.1002/mmce.20663 . hal-00734076

\section{HAL Id: hal-00734076 https://hal.science/hal-00734076}

Submitted on 20 Sep 2012

HAL is a multi-disciplinary open access archive for the deposit and dissemination of scientific research documents, whether they are published or not. The documents may come from teaching and research institutions in France or abroad, or from public or private research centers.
L'archive ouverte pluridisciplinaire HAL, est destinée au dépôt et à la diffusion de documents scientifiques de niveau recherche, publiés ou non, émanant des établissements d'enseignement et de recherche français ou étrangers, des laboratoires publics ou privés. 


\title{
A CAD TOOL FOR AN ARRAY OF DIFFERENTIAL OSCILLATORS COUPLED THROUGH A BROADBAND NETWORK
}

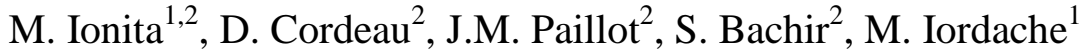 \\ ${ }^{1}$ Electrical Engineering Faculty, University Politehnica of Bucharest, 313 Splaiul Independentei, \\ 060042 Bucharest - Romania \\ ${ }^{2}$ LAII ENSIP, EA 1219, University of Poitiers, IUT, 4 Av de Varsovie, 16021 Angoulême - \\ France
}

Shortened title: A CAD TOOL FOR COUPLED OSCILLATORS

\begin{abstract}
A new expression of the equations describing the locked states of two oscillators coupled through a resistor is presented in this paper. This theory has led to the elaboration of a CAD tool which provides, in a short simulation time, the frequency locking region of two coupled differential oscillators.
\end{abstract}

Key words: broadband network, van der Pol differential oscillators, synchronization, coupled oscillators, design automation.

\section{Introduction}

Arrays of coupled oscillators are the subject of increasing research activity due to their use in new applications such as power-combining techniques or beam steering of antenna arrays, by synthesizing a constant phase shift distribution between two adjacent oscillators [1-6]. For a onedimensional oscillators array, the required inter-stage phase shift can be obtained by detuning the free-running frequencies of the outermost oscillators in the array [5]. Furthermore, it is shown in $[4,7,8]$ that the resulting phase shift is independent of the number of oscillators in the array. 
In R. York's previous works, coupled microwave oscillators have been modeled as simple single-ended van der Pol oscillators coupled through either a resistive network or a broadband network $[4,7,9]$. Unfortunately, these works are limited to the cases when the coupling network bandwidth is much wider than the oscillators' bandwidth. In these conditions, a generalization of Kurokawa's method [10] was used to extend the study to a narrow-band circuit allowing the equations for the amplitude and phase dynamics of two oscillators coupled through many types of circuits to be derived [11].

Since these works, only a small number of articles have presented new techniques for the analysis of coupled-oscillator arrays [12-15]. In [14], a semi-analytical formulation is proposed for the design of coupled-oscillator systems, avoiding the computational expensiveness of a full harmonic balance synthesis exposed in [12,13]. In [15], a simplified closed-form of the semianalytical formulation proposed in [14] for the optimized design of coupled-oscillator systems is presented. Nevertheless, even if these new semi-analytical formulations permit a good prediction of the coupled-oscillator solution, it is only valid for the weak coupling case. On the other hand, the York's theory [11] provides a full analytical formulation allowing to predict the performances of microwave oscillator arrays for both weak and strong coupling.

In the authors' previous work [16], mathematical manipulations were applied to the dynamic equations describing the locked states of the coupled oscillators proposed in [11]. A reduced system of equations was obtained, leading to the elaboration of a CAD tool that provides, in a considerably short simulation time, the frequency locking region of the coupled oscillators, in terms of the amplitudes of their output signals and the phase shift between them. Nevertheless, although this $\mathrm{CAD}$ tool allows to obtain, in a short simulation time, the cartography of the locked states of the coupled oscillators, it is limited to the case of two single-ended van der Pol 
oscillators coupled through an RLC circuit. Furthermore, it does not allow an accurate prediction of the oscillators' amplitudes since the van der Pol model used by J. Lynch and R. York in [11] is too simple.

Moreover, since the theoretical limit of the phase shift obtained for an array of single-ended coupled oscillators is within the range of $\pm 90^{\circ}$ [7], it seems interesting to analyze the behavior of an array of differential oscillators. Indeed, in this case, the theoretical limit of the phase shift is within $360^{\circ}$ due to the differential nature of the array, leading to a more efficient beam-scanning architecture for instance. Furthermore, differential oscillators are widely used in high-frequency circuit design due to their relatively good phase noise performances and ease of integration. Moreover, the use of a broadband coupling network, i.e. a resistor, instead of a resonant one, can lead to a substantial save in chip area.

Due to these considerations, the aim of this paper is to present a new CAD tool allowing to acquire, in a considerably short simulation time, the frequency locking region of two differential oscillators coupled through a resistive network.

This paper will be organized as follows. A brief review of R. York's theory giving the dynamics for two van der Pol oscillators coupled through a resonant network will be presented in subsection II-A. In subsection II-B, a new expression of the equations describing the locked states of two van der Pol oscillators coupled through a broadband network using an accurate model allowing a good prediction of the oscillators' amplitude will be presented. Then, in subsection II-C, we will introduce a reduced system of equations with no trigonometric aspects allowing an easier numerical solving method. In section III, the developed CAD tool as well as its reliability test using Agilent's ADS software will be presented. The details concerning the 
variables estimation technique used to solve the system of equations describing the locked states of the two van der Pol coupled oscillators as well as the stability analysis of the locked states will be described in subsections III-A and III-B, respectively. Finally, in subsection III-C, the simulation results showing the reliability and the accuracy of the proposed tool will be presented.

\section{Coupled van der Pol oscillators theory}

\section{A. Overview of R. York's theory}

R. York's work considers the analysis of simple single-ended van der Pol oscillators coupled through either a resistive network or a broadband network that produce a constant amplitude and a phase shift between the oscillators [7,9]. This analysis is useful in showing the effects of the coupling network parameters but, unfortunately, it is limited to the broadband case. In these conditions, R. York used a generalization of Kurokawa's method [10] to extend the oscillators dynamics to the case of narrow-band coupling. Using a system made of two parallel resonant circuits containing nonlinear negative conductance devices and coupled through a series resonant circuit, as shown in Fig. 1, and starting from the admittance transfer functions $\left(Y_{1}, Y_{2}, Y_{\mathrm{c}}\right)$ binding the coupling current $\left(I_{\mathrm{c}}\right)$ to the oscillators' voltages $\left(V_{1}\right.$ and $\left.V_{2}\right)$, J. Lynch and R. York described the oscillators' dynamic equations, as well as those for the amplitude and phase of the

coupling current. Then, by setting the derivatives to zero, the algebraic equations describing the oscillators' frequency locked states were obtained as follows: 


$$
\begin{gathered}
\left(1-\lambda_{0} \varepsilon^{2}-A_{1}^{2}\right) A_{1}=-\lambda_{0} \varepsilon A_{2} \cos (\Delta \phi-\Phi) \\
\Delta \omega_{01}-\left(1-\lambda_{0} \varepsilon \frac{\omega_{a}}{\omega_{a c}}\right) \Delta \omega_{c}=-\lambda_{0} \varepsilon \omega_{a} \frac{A_{2}}{A_{1}} \sin (\Delta \phi-\Phi) \\
\left(1-\lambda_{0} \varepsilon^{2}-A_{2}^{2}\right) A_{2}=-\lambda_{0} \varepsilon A_{1} \cos (\Delta \phi+\Phi) \\
\Delta \omega_{02}-\left(1-\lambda_{0} \varepsilon \frac{\omega_{a}}{\omega_{a c}}\right) \Delta \omega_{c}=\lambda_{0} \varepsilon \omega_{a} \frac{A_{1}}{A_{2}} \sin (\Delta \phi+\Phi)
\end{gathered}
$$

with:

- $\lambda_{0}=\frac{1}{G_{0} R_{c}}$ : the coupling constant, where $G_{0}$ is the nonlinear conductance at zero voltage;

- $2 \omega_{a}=\frac{G_{0}}{C}:$ the oscillator bandwidth;

- $2 \omega_{a c}=\frac{R_{c}}{L_{c}}$ : the unloaded coupling circuit bandwidth;

- $A_{1}, A_{2}$ : the amplitudes of oscillators 1 and 2, respectively;

- $\Delta \phi=\phi_{2}-\phi_{1}$ : the inter-stage phase shift;

- $\omega_{01}, \omega_{02}$ : the free-running frequencies or tunings of oscillators 1 and 2, respectively;

- $\omega_{0 c}$ : the resonant frequency of the coupling circuit;

$$
\text { - } \varepsilon=\frac{1}{\sqrt{1+\left(\frac{\Delta \omega_{c}}{\omega_{a c}}\right)^{2}}}=\cos (\Phi): \text { the coupling strength scale factor; }
$$


- $\Phi=\tan ^{-1}\left(\frac{\Delta \omega_{c}}{\omega_{a c}}\right):$ the coupling phase.

Furthermore, let us note that in the equations presented above, we refer the oscillators' freerunning frequencies or tunings, $\omega_{01}$ and $\omega_{02}$, and the synchronization frequency of the system, $\omega$, to the coupling circuit resonant frequency, $\omega_{0 c}$, using the following substitutions:

$$
\Delta \omega_{01}=\omega_{01}-\omega_{0 c} \quad \Delta \omega_{02}=\omega_{02}-\omega_{0 c} \quad \Delta \omega_{c}=\omega-\omega_{0 c}
$$

Thus, a solution to (1) indicates the existence of a frequency-locked state and allows to obtain the amplitudes $A_{1}$ and $A_{2}$ of the two oscillators as well as the inter-stage phase shift $\Delta \phi$ and the synchronization pulsation $\omega$ for a combination $\left(\Delta \omega_{01}, \Delta \omega_{02}\right)$.

\section{B. New expression of the equations allowing an accurate prediction of the oscillators' amplitudes}

The purpose of this subsection is to present a new formulation of York's equations describing the locked states of two coupled van der Pol oscillators using an accurate model allowing a good prediction of the oscillators' amplitudes. Indeed, in [11], the negative conductance presented by the active part has the following expression:

$$
-G(A)=-G_{0}\left(1-A^{2}\right)
$$

leading to a free-running amplitude of one van der Pol oscillator always equal to unity irrespective of the $G_{0}$ value. Thus, this result clearly shows the limitation of the van der Pol model used by Lynch and York in [11]. In this case, in order to provide a more accurate model, 
let us consider the schematic of a van der Pol oscillator as shown in Fig. 2, for which the current in the nonlinear conductance $G_{\mathrm{NL}}$ is equal to $i(t)=-a v(t)+b v^{3}(t)$ with $-a$ the negative conductance necessary to start the oscillations and $b$ a parameter used to model the saturation phenomenon. Now, assuming a perfectly sinusoidal oscillation so that $v(t)=A \cdot \cos \left(\omega_{0} t\right)$, the expression of the current $i(t)$ is the following:

$$
i(t)=\left(-a+\frac{3}{4} b A^{2}\right) \cdot A \cdot \cos \left(\omega_{0} t\right)+b \frac{A^{3}}{4} \cos \left(3 \omega_{0} t\right)
$$

Furthermore, let us recall that such an oscillator topology can be modeled by a quasi-linear representation allowing a very simple analytical calculation [17]. In this case, the expression in the first bracket in (3) represents the negative conductance presented by the active part.

Now, in order to obtain the new system of four equations describing the locked states of two coupled van der Pol oscillators, let us consider the circuit of Fig. 3 made of two van der Pol oscillators coupled through a series RLC circuit. In this case, the frequency-domain equations, using the admittance transfer function binding the coupling current to the oscillators' voltages, can be written as follows:

$$
\begin{gathered}
I_{c}=Y_{1}\left(A_{1}, \omega_{1}\right) \cdot V_{1}=\left[-G_{N L}\left(A_{1}\right)+Y_{L 1}\left(\omega_{1}\right)\right] \cdot V_{1} \\
I_{c}=-Y_{2}\left(A_{2}, \omega_{2}\right) \cdot V_{2}=-\left[-G_{N L}\left(A_{2}\right)+Y_{L 2}\left(\omega_{2}\right)\right] \cdot V_{2} \\
I_{c}=-Y_{c}\left(\omega_{c}\right) \cdot\left(V_{1}-V_{2}\right)
\end{gathered}
$$

where $A_{1}, \omega_{1}$, represents the amplitude and pulsation of oscillator 1 , and so on and the subscripts " $N L "$ and " $L "$ denote respectively the nonlinear and linear portions of the circuit. The admittance 
transfer function for oscillator 1, assuming that the frequency of the oscillator remains close to its "free running" or uncoupled value $\omega_{01}$, has the following expression:

$$
Y_{1}\left(A_{1}, \omega_{1}\right) \cong G_{L}\left(1-\frac{G_{N L}\left(A_{1}\right)}{G_{L}}\right)+\frac{2 C}{j}\left(\omega_{01}-\omega_{1}\right)
$$

where $\omega_{01}=\frac{1}{\sqrt{L_{1} C}}$ is the tank resonant frequency of oscillator 1 and $-G_{N L}\left(A_{1}\right)=-a+\frac{3}{4} b A_{1}{ }^{2}$ is the negative conductance presented by the active part as expressed in (3) leading to sinusoidal oscillation.

Now, let us note $2 \omega_{a}=\frac{G_{L}}{C}$ which represents the resonator "bandwidth" and using the substitution $\frac{G_{N L}\left(A_{1}\right)}{G_{L}}-1=S\left(A_{1}\right)$ in order to simplify the notation, the expressions for the admittances of the oscillator 1 and 2, are respectively:

$$
\begin{aligned}
& Y_{1}\left(A_{1}, \omega_{1}\right) \cong-G_{L}\left[S\left(A_{1}\right)+j \frac{\left(\omega_{01}-\omega_{1}\right)}{\omega_{a}}\right] \\
& Y_{2}\left(A_{2}, \omega_{2}\right) \cong-G_{L}\left[S\left(A_{2}\right)+j \frac{\left(\omega_{02}-\omega_{2}\right)}{\omega_{a}}\right]
\end{aligned}
$$

Thus, following the same steps as in [11] but using (6) and (7) for the admittance functions of oscillators 1 and 2 and replacing $G_{0}$ by $G_{L}$, the new system of equations describing the locked states of two van der Pol oscillators coupled through a resonant network is the following: 


$$
\begin{aligned}
& \left(S\left(A_{1}\right)-\lambda_{0} \varepsilon^{2}\right) A_{1}=-\lambda_{0} \varepsilon A_{2} \cos (\Delta \phi-\Phi) \\
& \Delta \omega_{01}-\left(1-\lambda_{0} \varepsilon \frac{\omega_{a}}{\omega_{a c}}\right) \Delta \omega_{c}=-\lambda_{0} \varepsilon \omega_{a} \frac{A_{2}}{A_{1}} \sin (\Delta \phi-\Phi) \\
& \left(S\left(A_{2}\right)-\lambda_{0} \varepsilon^{2}\right) A_{2}=-\lambda_{0} \varepsilon A_{1} \cos (\Delta \phi+\Phi) \\
& \Delta \omega_{02}-\left(\begin{array}{cc}
1-\lambda_{0} \varepsilon & \frac{\omega_{a}}{\omega_{a c}}
\end{array}\right) \Delta \omega_{c}=\lambda_{0} \varepsilon \omega_{a} \frac{A_{1}}{A_{2}} \sin (\Delta \phi+\Phi)
\end{aligned}
$$

where $\lambda_{0}=\frac{1}{G_{L} R_{c}}, 2 \omega_{a}=\frac{G_{L}}{C}$ and $S\left(A_{i}\right)=\frac{G_{N L}\left(A_{i}\right)}{G_{L}}-1=\frac{a-\frac{3}{4} b A_{i}^{2}-G_{L}}{G_{L}}$.

The case of a resistive coupling circuit can be deduced from (8) by letting the frequency bandwidth of the unloaded coupling circuit approach infinity, so that $\omega_{a c} \rightarrow \infty$ leading to $\varepsilon=1$ and $\Phi=0$. In these conditions, since $\Delta \omega_{01}-\Delta \omega_{c}=\omega_{01}-\omega$ and $\Delta \omega_{02}-\Delta \omega_{c}=\omega_{02}-\omega$, the equations describing the locked states can be written as follows:

$$
\begin{gathered}
\left(S\left(A_{1}\right)-\lambda_{0}\right) A_{1}=-\lambda_{0} A_{2} \cos (\Delta \phi) \\
\omega_{01}=\omega-\lambda_{0} \omega_{a} \frac{A_{2}}{A_{1}} \sin (\Delta \phi) \\
\left(S\left(A_{2}\right)-\lambda_{0}\right) A_{2}=-\lambda_{0} A_{1} \cos (\Delta \phi) \\
\omega_{02}=\omega+\lambda_{0} \omega_{a} \frac{A_{1}}{A_{2}} \sin (\Delta \phi)
\end{gathered}
$$

\section{New expression of the equations describing the locked states of two van der Pol oscillators coupled through a resistor}

Once more, a solution to (9) indicates the existence of a frequency-locked state for the two van der Pol coupled oscillators. However, in this case, one can obtain the oscillators' amplitudes $A_{1}$ 
and $A_{2}$ for any values of parameters $a$ and $b$ of the van der Pol non linearity under a sinusoidal assumption as well as the inter-stage phase shift $\Delta \phi$ and the synchronization pulsation $\omega$ for a combination $\left(\omega_{01}, \omega_{02}\right)$.

Nevertheless, due to the trigonometric and nonlinear aspect of equations (9), the solutions of this system are very difficult or impossible to obtain. In these conditions, mathematical manipulations are applied to the equations in order to obtain a new system of three equations with three unknowns $A_{1}, A_{2}$ and $\omega$, easier to solve. Indeed, from (9) we can write:

$$
\begin{gathered}
\cos (\Delta \phi)=-\frac{\left(S\left(A_{1}\right)-\lambda_{0}\right) A_{1}}{\lambda_{0} A_{2}} \\
\sin (\Delta \phi)=-\frac{\omega_{01}-\omega}{\lambda_{0} \omega_{a} \frac{A_{2}}{A_{1}}} \\
\cos (\Delta \phi)=-\frac{\left(S\left(A_{2}\right)-\lambda_{0}\right) A_{2}}{\lambda_{0} A_{1}} \\
\sin (\Delta \phi)=\frac{\omega_{02}-\omega}{\lambda_{0} \omega_{a} \frac{A_{1}}{A_{2}}}
\end{gathered}
$$

Since $\cos ^{2}(\Delta \phi)+\sin ^{2}(\Delta \phi)=1$, we have:

$$
\left\lceil\frac{\left(S\left(A_{1}\right)-\lambda_{0}\right) A_{1}}{\lambda_{0} A_{2}}\right]^{2}+\left|\frac{\omega_{01}-\omega}{\lambda_{0} \omega_{a} \frac{A_{2}}{A_{1}}}\right|^{2}=1
$$

and 


$$
\left\{\left.\frac{\left(S\left(A_{2}\right)-\lambda_{0}\right) A_{2}}{\lambda_{0} A_{1}}\right|^{2}+\mid \frac{\omega_{02}-\omega}{\lambda_{0} \omega_{a} \frac{A_{1}}{A_{2}}}\right)^{2}=1
$$

Furthermore, according to (10), we obtain:

$$
\frac{\sin (\Delta \phi)}{\cos (\Delta \phi)}=\frac{\omega_{01}-\omega}{\omega_{a}\left(S\left(A_{1}\right)-\lambda_{0}\right)}
$$

and

$$
\frac{\sin (\Delta \phi)}{\cos (\Delta \phi)}=-\frac{\omega_{02}-\omega}{\omega_{a}\left(S\left(A_{2}\right)-\lambda_{0}\right)}
$$

Now using the substitution $X_{1}\left(A_{1}, \omega\right)=\frac{\omega_{01}-\omega}{\omega_{a}\left(S\left(A_{1}\right)-\lambda_{0}\right)}$ and $X_{2}\left(A_{2}, \omega\right)=\frac{\omega_{02}-\omega}{\omega_{a}\left(S\left(A_{2}\right)-\lambda_{0}\right)}$,

then (11) and (12) can be written as:

$$
\begin{aligned}
& A_{1}^{2}\left(S\left(A_{1}\right)-\lambda_{0}\right)^{2}\left(1+X_{1}^{2}\left(A_{1}, \omega\right)\right)=\lambda_{0}^{2} A_{2}^{2} \\
& A_{2}^{2}\left(S\left(A_{2}\right)-\lambda_{0}\right)^{2}\left(1+X_{2}^{2}\left(A_{2}, \omega\right)\right)=\lambda_{0}^{2} A_{1}^{2}
\end{aligned}
$$

In the same way, (13) and (14) can be expressed as:

$$
\tan (\Delta \phi)=X_{1}\left(A_{1}, \omega\right)
$$




$$
\tan (\Delta \phi)=-X_{2}\left(A_{2}, \omega\right)
$$

And dividing (17) and (18) yields:

$$
-\frac{X_{1}\left(A_{1}, \omega\right)}{X_{2}\left(A_{2}, \omega\right)}=1 \Rightarrow X_{1}\left(A_{1}, \omega\right)+X_{2}\left(A_{2}, \omega\right)=0
$$

Hence, the new system of three equations with three unknowns $A_{1}, A_{2}$ and $\omega$, is presented as shown below:

$$
\begin{gathered}
A_{1}^{2}\left(S\left(A_{1}\right)-\lambda_{0}\right)^{2}\left(1+X_{1}^{2}\left(A_{1}, \omega\right)\right)=\lambda_{0}^{2} A_{2}^{2} \\
A_{2}^{2}\left(S\left(A_{2}\right)-\lambda_{0}\right)^{2}\left(1+X_{2}^{2}\left(A_{2}, \omega\right)\right)=\lambda_{0}^{2} A_{1}^{2} \\
X_{1}\left(A_{1}, \omega\right)+X_{2}\left(A_{2}, \omega\right)=0
\end{gathered}
$$

where:

$$
\begin{aligned}
& X_{1}\left(A_{1}, \omega\right)=\frac{\omega_{01}-\omega}{\left(S\left(A_{1}\right)-\lambda_{0}\right) \omega_{a}} \\
& X_{2}\left(A_{2}, \omega\right)=\frac{\omega_{02}-\omega}{\left(S\left(A_{2}\right)-\lambda_{0}\right) \omega_{a}}
\end{aligned}
$$

Once this system of equations is solved, the solutions will then allow to determine the phase shift $\Delta \phi$ between the two adjacent elements according to the pulsations $\omega_{01}$ and $\omega_{02}$ using the following expressions:

$$
\Delta \phi=\arctan \left(X_{1}\left(A_{1}, \omega\right)\right)
$$

or 


$$
\Delta \phi=\arctan \left(-X_{2}\left(A_{2}, \omega\right)\right)
$$

\section{CAD Tool}

In this section, the new CAD tool which allows to obtain, in a considerably short simulation time, the cartography of the locked states of two differential oscillators coupled through a resistive network is presented. Hence, the system of equations (20) was implemented on Matlab and solved using nonlinear programming technique.

\section{A. Variables estimation technique}

In this part, the problem of identifying the variables (or the roots) of the system of equations (20) is discussed. The basic formulation of the general problem is the following: given $a n^{\text {th }}$ dimension system of nonlinear coupled functions $f_{k}(\underline{\theta})$, we seek the value of the variables' vector $\underline{\theta}$ for which $\left(f_{k}(\underline{\theta})=0, k=1, n\right)$.

In the case of the two van der Pol coupled oscillators model given previously, a new root finding procedure based on parameter estimation technique is developed. Let us consider the previous mathematical model given by equations (20). The vector of variables to be estimated is:

$$
\underline{\theta}=\left[\begin{array}{lll}
A_{1}^{2} & A_{2}^{2} & \frac{\omega}{\omega_{a}}
\end{array}\right]^{T}
$$

Where [.] ${ }^{T}$ denotes a transposition operation.

Furthermore, the nonlinear coupled functions $f_{k}(\underline{\theta})$ are found from (20) as: 


$$
\begin{array}{r}
f_{1}(\underline{\theta})=A_{1}^{2}\left[\left(a-\frac{3}{4} b A_{1}^{2}-G_{L}\left(1+\lambda_{0}\right)\right)^{2}+G_{L}^{2}\left(\frac{\omega_{01}}{\omega_{a}}-\frac{\omega}{\omega_{a}}\right)^{2}\right]-\lambda_{0}^{2} A_{2}^{2} G_{L}^{2} \\
f_{2}(\underline{\theta})=A_{2}^{2}\left[\left(a-\frac{3}{4} b A_{2}^{2}-G_{L}\left(1+\lambda_{0}\right)\right)^{2}+G_{L}^{2}\left(\frac{\omega_{02}}{\omega_{a}}-\frac{\omega}{\omega_{a}}\right)^{2}\right]-\lambda_{0}^{2} A_{1}^{2} G_{L}^{2} \\
f_{3}(\underline{\theta})=\left(\frac{\omega_{01}}{\omega_{a}}-\frac{\omega}{\omega_{a}}\right)\left(a-\frac{3}{4} b A_{2}^{2}-G_{L}\left(1+\lambda_{0}\right)\right)+\left(\frac{\omega_{02}}{\omega_{a}}-\frac{\omega}{\omega_{a}}\right)\left(a-\frac{3}{4} b A_{1}^{2}-G_{L}\left(1+\lambda_{0}\right)\right)
\end{array}
$$

As a general rule, estimation with Output Error technique $[18,19]$ is based on minimization of a quadratic multivariable criterion defined as :

$$
J=\sum_{k=1}^{3} f_{k}(\underline{\theta})^{2}
$$

Unlike linear equations, most of the nonlinear equations cannot be solved in a finite number of steps. Iterative methods are being used to solve nonlinear equations. For criterion minimization, the variables values $\underline{\theta}$ can be driven iteratively to the optimum by Non Linear Programming techniques. Practically, we are using Marquardt's algorithm [20] for off-line estimation:

$$
\underline{\hat{\theta}}_{i+1}=\underline{\hat{\theta}}_{i}-\left\{\left[J_{\theta \theta}^{\prime \prime}+\lambda . I\right]^{-1} \cdot J_{\theta}^{\prime}\right\}_{\underline{\hat{\theta}}=\underline{\theta}_{i}}
$$

where

$\hat{\theta}$ is an estimation of the system solutions $\underline{\theta}$, 
$J_{\theta}^{\prime}=\frac{\partial J}{\partial \underline{\theta}}=-2 \cdot \sum_{k=1}^{3} f_{k} \cdot \underline{\sigma}_{k, \underline{\theta}}$ is the gradient of criterion,

$J_{\theta \theta}^{\prime \prime}=\frac{\partial^{2} J}{\partial \underline{\theta}^{2}} \approx 2 \cdot \sum_{k=1}^{3} \underline{\sigma}_{k, \underline{\theta}} \cdot \underline{\sigma}_{k, \underline{\theta}}^{T}$ is the hessian,

$\lambda$ is the monitoring parameter,

and $\underline{\sigma}_{k, \underline{\theta}}=\frac{\partial f_{k}}{\partial \underline{\theta}}$ is the output sensitivity function or derivative of $f_{k}(\underline{\theta})$ according to $\underline{\theta}$.

It is important to note that the obtained $\underline{\hat{\theta}}$ is an approximated solution if $\mathrm{J} \approx 0$, i.e. $f_{k}(\underline{\theta}) \approx 0, k=1,3$. This final value of the criterion corresponds to "small residual".

\section{B. Stability analysis}

For the stability analysis of the synchronized states, it is important to consider an initial differential system describing the amplitudes $\left(A_{1}, A_{2}\right)$ and phases $\left(\phi_{1}, \phi_{2}\right)$ dynamics and the coupling current in Cartesian format $\left(A_{c x}, A_{c y}\right)$. Thus, dynamic equations developed in [11] have been adapted to the studied case and give the following differential relations:

$$
\underline{x}=\underline{f}\left(\underline{x}, \omega_{a}, \omega_{01}, \omega_{02}\right)
$$

where

$\underline{x}=\left[\begin{array}{llllll}A_{1} & \phi_{1} & A_{2} & \phi_{2} & A_{c x} & A_{c y}\end{array}\right]^{T}$ 
and

$$
\underline{f}\left(\underline{x}_{a}, \omega_{01}, \omega_{02}\right)=\left\{\begin{array}{l}
\omega_{a} A_{1}\left|\frac{a-\frac{3}{4} b A_{1}^{2}-G_{L}}{G_{L}}\right|+\omega_{a}\left(A_{c x} \cos \left(\phi_{1}\right)+A_{c y} \sin \left(\phi_{1}\right)\right) \\
\left.\omega_{01}-\omega-\frac{\omega_{a}}{A_{1}} \mid A_{c x} \sin \left(\phi_{1}\right)-A_{c y} \cos \left(\phi_{1}\right)\right] \\
\omega_{a} A_{2}\left|\frac{a-\frac{3}{4} b A_{2}-G_{L}}{G_{L}}\right|-\omega_{a}\left(A_{c x} \cos \left(\phi_{2}\right)+A_{c y} \sin \left(\phi_{2}\right)\right) \\
\omega_{02}-\omega-\frac{\omega_{a}}{A_{2}}\left[A_{c x} \sin \left(\phi_{2}\right)-A_{c y} \cos \left(\phi_{2}\right)\right] \\
-\omega_{a c} A_{c x}+\left(\omega-\omega_{0 c}\right) A_{c y}+\omega_{a c} \lambda_{0}\left[A_{2} \cos \left(\phi_{2}\right)-A_{1} \cos \left(\phi_{1}\right)\right] \\
-\omega_{a c} A_{c y}-\left(\omega-\omega_{0 c}\right) A_{c x}+\omega_{a c} \lambda_{0}\left[A_{2} \sin \left(\phi_{2}\right)-A_{1} \sin \left(\phi_{1}\right)\right]
\end{array}\right]
$$

with

$A_{c x}=\frac{I_{c}}{G_{L}} \cos \left(\phi_{c}\right), A_{c y}=\frac{I_{c}}{G_{L}} \sin \left(\phi_{c}\right)$ and $\phi_{c}$ is the phase of the coupling current.

These differential equations are nonlinear in states $\underline{x}$, and unfortunately, the stability theory developed for the linear problem does not apply directly to this system. In practice, we typically linearize this system around a synchronized solution noted $\underline{x}_{0}$ and consider the eigenvalues of the Jacobian matrix $[A]$ so that:

$$
\dot{\delta x}=\left[\frac{\partial \underline{f}}{\partial \underline{x}}\right]_{\underline{x}=\underline{x}_{0}} . \underline{\delta x}=[A] \cdot \underline{\delta x}
$$


where $\underline{\delta x}=\underline{x}-\underline{x}_{0}$ are the small variations of the synchronized states $\underline{x}$ and $[A]^{6 \times 6}=\left[\frac{\partial f}{\partial \underline{x}}\right]_{\substack{x=x_{0}\\}}$ is

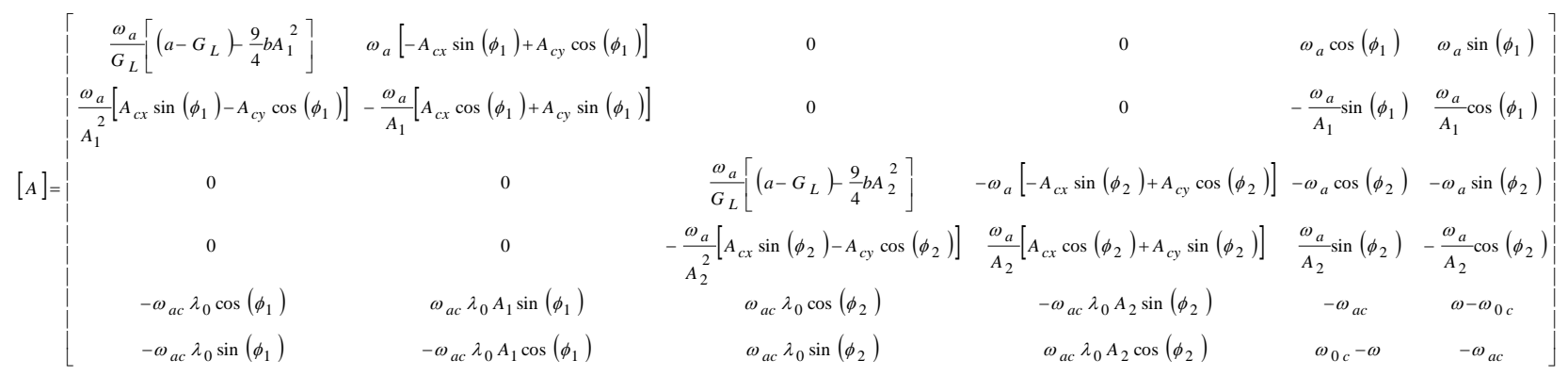

Synchronized states are asymptotically stable if and only if all the eigenvalues of the Jacobian matrix $[A]$ have negative real parts. Thus, after each estimation of variables values $\underline{\theta}$, the resulting Jacobian matrix is evaluated and these six eigenvalues, noted $\mu_{i}$, are computed. Hence, the obtained solution is asymptotically stable if all eigenvalues $\mu_{i}$ of the Jacobian matrix $[A]$ satisfy $\mathfrak{R}$ eal $\left(\mu_{i}\right)<0$ whereas the solution point is unstable if at least one eigenvalue $\mu_{i}$ satisfies $\mathfrak{R}$ eal $\left(\mu_{i}\right) \geq 0$.

Let us note that in the developed CAD tool, the eigenvalues are obtained using the MATLAB software function $e i g(\cdot)$.

\section{Simulation results}

In order to show the accuracy and the reliability of the proposed CAD tool, simulations using Agilent's ADS software will be performed. In these conditions, let us consider the design of 
two identical differential oscillators coupled through a resistor $R_{c} / 2$ of $200 \Omega$, as shown in Fig. 4. The oscillators' structure is based on a cross-coupled NMOS differential topology using a 0.35 $\mu \mathrm{m}$ BiCMOS SiGe process. The cross connected NMOS differential pair provides the negative resistance to compensate for the tank losses, and the tail current source is a simple NMOS current mirror which draws $28 \mathrm{~mA}$. The frequency of oscillation is chosen to be close to $6 \mathrm{GHz}$ and is determined by the $L C$ tank at the drains. In these conditions, the inductance value, $L_{1,2}$, is close to $0.8 \mathrm{nH}$ and the capacitor value, $C$ is close to $0.88 \mathrm{pF}$. The resistor value, $R$, is equal to $100 \Omega$ so that the quality factor of the tank is equal to 3.3 . A tail capacitor $C_{T}$ is used to attenuate both the high-frequency noise component of the tail current and the voltage variations on the tail node [21]. To ensure proper start-up of the oscillator, the transconductance of the NMOS transistor should be greater than $\frac{1}{R}$. In these conditions, the sizes of NMOS transistors $T_{1}$ to $T_{4}$ are identical and chosen to be $\left(\frac{W}{L_{g}}\right)=\frac{70 \mu m}{0.35 \mu m}$.

Since the presented theory implemented in our CAD tool uses van der Pol oscillators to model microwave coupled oscillators, we performed the modeling of this structure as two differential van der Pol coupled oscillators as presented in [22], using ADS simulation results for one differential NMOS oscillator at the required synchronization frequency. As a consequence, the two coupled oscillators of Fig. 4 can be reduced into two differential van der Pol coupled oscillators as shown in Fig. 5. In this case, let us note that the value of the coupling resistor on each path is equal to $R c / 2$ to match well with the theory based on the use of two single-ended van der Pol oscillators. For the modelling of the active part, the $I=f\left(V d_{1^{-}}-V d_{2}\right)$ characteristic of one differential NMOS oscillator of Fig.4 at the required synchronization frequency was plotted leading to the typical cubic nonlinearity of a van der Pol oscillator. Hence, from this 
characteristic, the values of parameters $a$ and $b$ of the negative conductance presented by the active part of each oscillator were found to be respectively equal to $7.55 \circ 10^{-3}$ and 4 ॰ $10^{-4}$.

Then, knowing the parameters $\lambda_{0}, \omega_{\mathrm{a}}, a$ and $b$, the proposed CAD tool provides the cartography of the locked states of the two differential coupled oscillators. For instance, for a synchronization frequency of $5.97 \mathrm{GHz}$, with $\omega_{a}=5.68 \odot 10^{9} \mathrm{rad} / \mathrm{s}$ and a coupling constant $\lambda_{0}=0.5$, the cartography of the oscillators' locked states provided by the CAD tool is presented in Fig. 6 . Thus, this figure presents the variations of the phase shift, $\Delta \phi$, the oscillators' amplitudes $A_{1}$ and $A_{2}$, and the synchronization frequency $f_{s}$ in function of $f_{01}$ and $f_{02}$.

In order to validate the results provided by our CAD tool, we compared them to the simulation results of the two coupled differential oscillators of Fig.4, obtained with Agilent's ADS software. Let us note that with ADS, only a transient analysis of one point at a time of synchronization region allows to verify the synchronization results obtained with the CAD tool. The amplitudes of the oscillators' output voltages as well as the phase shift between them and the synchronization frequency are provided for each combination of $f_{01}$ and $f_{02}$. For instance, let us now consider Fig. 6 where the point marked with an arrow in the four subplots represents a freerunning frequency $f_{01}=5.73 \mathrm{GHz}$ for oscillator 1 and $f_{02}=6.21 \mathrm{GHz}$ for oscillator 2 . Let us note that these two free-running frequencies correspond to the equally spaced tunings line as presented in [11]. The marked points lead to a phase shift of $32.39^{\circ}$, a synchronization frequency of the coupled oscillators of $5.97 \mathrm{GHz}$ and an amplitude of $2.68 \mathrm{~V}$ at the output of each of the coupled oscillators.

In the same conditions, the two differential NMOS coupled oscillators of Fig.4 simulated with ADS have lead to two sinusoidal waves at a synchronization frequency of $5.98 \mathrm{GHz}$, a phase 
shift of $31.23^{\circ}$ and an amplitude of $2.72 \mathrm{~V}$ at the output of each oscillator, as presented in Fig. 7. Thus, the errors regarding the phase shift and the amplitude predictions are only $3.71 \%$ and 1.49 $\%$, respectively.

Furthermore, Table I shows a comparison between the synchronization frequency, the phase shift and the amplitudes obtained in simulations with ADS for the two coupled NMOS differential oscillators, and with our CAD tool. Let us note that the free-running frequencies chosen for this table correspond to the equally spaced tunings line for which the synchronization frequency is equal to $5.97 \mathrm{GHz}$. Table II shows the same comparison as Table I, except that the free-running frequencies are not referred to the equally spaced tunings line. Hence, a good agreement was found between the results presented in both tables showing the reliability and the accuracy of the presented CAD tool. Nevertheless, as we approach the locking-region boundary, one can observe that the difference between the theoretical and simulated results is increasing. This is mainly due to the fact that the modeling of each NMOS differential oscillator as a differential van der Pol oscillator has been performed only at one frequency, i.e. the desired synchronization frequency.

Anyway, these results show that it is possible to adjust, with a relatively high accuracy, the freerunning frequencies of the two differential NMOS oscillators required to achieve the desired phase shift, amplitudes and synchronization frequency. The main advantage of this CAD tool is that, in an extremely short simulation time, one can obtain all the phase shifts, synchronization frequencies and amplitudes of the differential coupled system, in function of the free-running frequencies $f_{01}$ and $f_{02}$ of the two differential oscillators. Hence, since the inter-stage phase shift is independent of the number of oscillators in the array $[4,7,9]$, the proposed tool can also help the designer to rapidly find the free-running frequencies of the two outermost oscillators of the array required to achieve the desired phase shift. Indeed, without such a tool, a transient analysis 
of the oscillator array for different couples of oscillators' free-running frequencies must be performed in order to find the phase shift required for the targeted application. The proposed CAD tool can of course be used for other differential oscillators architectures and in strong and weak coupling cases.

\section{Conclusion}

This paper presents a new CAD tool which provides, in a considerably shorter analysis time a cartography giving the phase shifts, synchronization frequencies and amplitudes of the differential output voltages of two differential oscillators coupled through a broadband network. Starting from a new expression of the equations describing the locked states of two van der Pol oscillators coupled through a resistor, a simpler system of three equations with three unknowns was obtained. Solving this system on Matlab using nonlinear programming technique has led to the elaboration of the cartography of the locked states of the two differential oscillators coupled through a resistive network.

The reliability and the accuracy of the developed CAD tool was verified using Agilent's ADS software, where two differential NMOS oscillators coupled through a resistor were simulated. The obtained results were in accordance with those generated by the presented CAD tool, showing the usefulness of such a tool for the design of an array of differential oscillators coupled through a broadband network.

\section{References}

[1] M. R. Kuhn and E. M. Biebl, "Power combining by means of harmonic injection locking", IEEE MTT-S Int. Microw. Symp. Dig, vol. 1, pp. 91-94, June 2004. 
[2] S. Nogi, J. Lin, and T. Itoh, "Mode analysis and stabilization of a spatial power combining array with strongly coupled oscillators", IEEE Transactions on Microwave Theory and Techniques, vol. 41, ${ }^{\circ}$ 10, pp. 1827-1837, October 1993.

[3] T. Health, "Beam steering of nonlinear oscillator arrays through manipulation of coupling phases", IEEE Transactions on Antennas and Propagation, vol. 52, n 7, pp. 1833-1842, July 2004.

[4] P. Liao and R. A. York, "Beam Scanning With Coupled VCOs", In Proc. Antennas Propagation Soc. Int. Symp., vol. 2, pp. 836-839, June 1994.

[5] P. Liao and R.A. York, "A new phase-shifterless beam-scanning technique using arrays of coupled oscillators", IEEE Transactions Microwave theory and techniques, vol. 41, n¹0, pp. 1810-1815, October 1993.

[6] S. Toon, A. Banai, F. Farzaneh, "Evaluation of beam steering in circular planar array of coupled microwave oscillators", International Journal of $R F$ and Microwave Computer-Aided Engineering, vol. 21, n4, pp. 383-391, July 2011.

[7] R. A. York, "Nonlinear analysis of phase relationships in quasioptical oscillator arrays", IEEE Transactions on Microwave Theory and Techniques, vol. 41, nº10, pp. 1799-1809, October 1993.

[8] R. A. York and T. Itoh, "Injection and phase-locking techniques for beam control", IEEE Transactions on Microwave Theory and Techniques, vol. 46, pp. 1920-1929, November 1998.

[9] R. A. York and P. Liao, "Oscillator Array Dynamics with Broadband N-Port Coupling Networks", IEEE Transactions on Microwave Theory and Techniques, vol. 42, $\mathrm{n}^{\circ}$ 11, pp. 2040-2045, November 1994.

[10] K. Kurokawa, "Injection locking of solid state microwave oscillators", In proc. IEEE, vol. 61, n¹0, pp. 1386-1409, October 1973.

[11] J. J. Lynch and R. A. York, "Synchronization of Oscillators Coupled Through Narrow-Band Networks", IEEE Transactions on Microwave Theory and Techniques, vol. 49, pp. 237-249, February 2001. 
[12] A. Collado, F. Ramirez, A. Suarez and J. P. Pascual, "Harmonic-Balance Analysis and Synthesis of Coupled-Oscillator Arrays", IEEE Microwave and Wireless Components Letters, vol. 14, pp. 192194, May 2004.

[13] A. Suarez, A. Collado and F. Ramirez, "Harmonic-Balance techniques for the design of coupledoscillator systems in both unforced and injection-locked operation", IEEE MTT-S Int. Microw. Symp. Dig, pp. 887-890, June 2005.

[14] A. Georgiadis, A. Collado and A. Suarez, "New Techniques for the Analysis and Design of CoupledOscillator Systems”, IEEE Transactions on Microwave Theory and Techniques, vol. 54, $\mathrm{n}^{\circ} 11$, pp. 3864-3877, November 2006.

[15] A. Suarez, F. Ramirez and S. Sancho, "Stability and Noise Analysis of Coupled-Oscillator Systems", IEEE Transactions on Microwave Theory and Techniques, vol. 59, n4, pp. 1032-1046, April 2011.

[16] N. Y. Tohmé, J.M. Paillot, D. Cordeau, P. Coirault, "Analysis of the Frequency Locking Region of Coupled Oscillators Applied to 1-D Antenna Arrays", European Microwave Conference, Amsterdam, Holland, pp. 1334-1337, October 2008.

[17] M. Odyniec, "RF and microwave oscillator design”, Boston, MA: Artech House; 2002.

[18] L. Ljung, "System identification: Theory for the user", 2nd edition, Prentice Hall, USA, 1999.

[19] S. Bachir and C. Duvanaud, "New Identification Procedure for Continuous-Time Radio Frequency Power Amplifier Model”, Journal of Circuits, Systems and Computers (JCSC), vol. 19, nº, pp. 1259-1274, October 2010.

[20] D.W. Marquardt, "An Algorithm for least-squares estimation of non-linear parameters”, Soc. Indust. Appl. Math, vol. 11, n², pp. 431-441, 1963.

[21] A. Hajimiri and T. H. Lee, "Design Issues in CMOS Differential LC Oscillators", IEEE Journal of Solid-State Circuits, vol. 34, no. 5, pp. 717-724, May 1999.

[22] M.I. Ionita, D. Cordeau, J.M. Paillot, M. Iordache, "Analysis and Design of an Array of Two Differential Oscillators Coupled Through a Resistive Network", European Conference on Circuit Theory and Design, Linköping, Sweden, pp. 73-76, August 2011. 


\section{Author's biography}

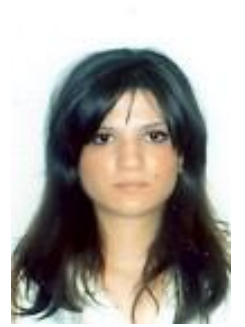

antenna arrays.

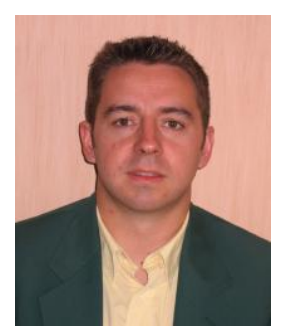

David Cordeau, PhD received the M.S. degree in electronics from the Ecole Nationale Supérieure d'Ingénieurs de Limoges, France in 2000 and the Ph.D. degree from the University of Poitiers, France, in 2004. His doctoral dissertation concerned the behavioral study and design of integrated polyphases VCOs using $\mathrm{Si}$ and SiGe technologies for radio communications.

From 2003 to 2005, he was with ACCO, Louveciennes, France, where he was in charge of the design of silicon RF integrated circuits. He joined the University of Poitiers, France, in 2005 as an assistant Professor and became an Associate Professor in 2006. His present research interests include RF and Microwave integrated circuits with an emphasis on VCOs and arrays of coupled oscillators. He has published several papers in international journals (IEEE JSSC, AEÜ IJEC, IET Microw.Antenn.Prop) and conferences (RFIC, IMS, ECWT, CSICS, ISCAS, NEWCAS, ECCTD). He has also one invited paper (ECAI'11) and one French patent.

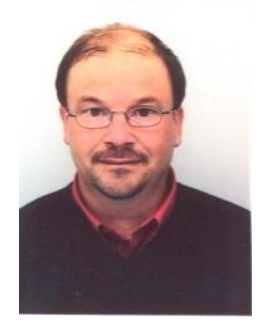

Jean-Marie Paillot received a PhD in Electronics form the University of Limoges, France, in 1990. His thesis on the design of non-linear analog circuits and the study of the noise spectra of integrated oscillators was prepared at the Institute IRCOM, Limoges, Frances.

After graduation, he joined the Electronics Laboratory of PHILIPS Microwave, at Limeil, France, as R\&D engineer in charge of the design of analog and numerical microwave monolithic integrated circuits. In October 1992, he joined the University of Poitiers, France, where he is currently Professor of Electronics Engineering.

Prof. J.M. Paillot is in charge of several contracts with industry and author of papers published in scientific journals, he is presently interested in phase noise reduction techniques for microwave oscillators (IEEE JSSC, AEÜ IJEC, 
RFIC, EuMC) and analysis of RF switched capacitor filters (RFIC, EuMC), as well as in phased-array transmitters (IET Microw.Antenn.Propag., IMS).

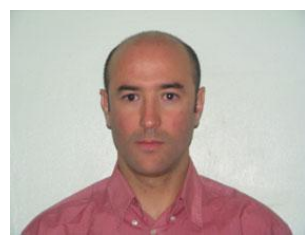

Smail Bachir, PhD received both his degree in Signal Theory from Polytechnic school of Algeria in 1997. He joined the scientific department of Leroy Somer Society and University of Poitiers in france where he received his Ph.D. degree in Automatic and Electrical

Engineering in 2002. He is presently an Associate Professor at the university of Poitiers and a researcher in the department of XLIM/SIC of University of Poitiers. His research interest include signal processing, parameter identification, telecommunication and radiocommunication circuits.

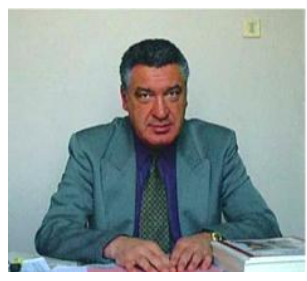

Prof. Eng. Mihai IORDACHE, PhD received the M.S. and Ph.D. degrees in electrical engineering from the Politehnica University of Bucharest, Romania, in 1967 and 1977, respectively. He is a Full Professor in the Electrical Department, Politehnica University of Bucharest, where he is working in the areas of circuit analysis and simulation, and in the Electrical Engineering Fundamentals. He is Doctoral Advisor at the Politehnica University of Bucharest, and the author or coauthor of more than 190 journal papers and 30 books. He is also a reviewer of different Scientific Conferences in the Analysis and Simulation Circuits. He was the recipient of the 2000 Romanian Academy Award.

\section{Figure Captions}

Fig. 1 - Two single-ended van der Pol oscillators coupled through a resonant circuit

Fig. 2 - The van der Pol oscillator model

Fig. 3 - Two van der Pol oscillators coupled through a RLC circuit

Fig. 4 - Two differential NMOS oscillators coupled through a resistor

Fig. 5 - Two differential van der Pol oscillators coupled through a resistor 
Fig. 6 - Cartography of the oscillators' locked states provided by the CAD tool

Fig. 7 - Waveforms of the output voltages of the two coupled differential NMOS oscillators, when $\Delta \phi=31.23^{\circ}$ and $A=2.72 \mathrm{~V}$

Table I - Comparison of the obtained results between ADS and the CAD tool for the case when $f_{01}$ and $f_{02}$ correspond to the equally spaced tunings line

Table II - Comparison of the obtained results between ADS and the CAD tool for the case when $f_{01}$ and $f_{02}$ aren't referred to the equally spaced tunings line

Fig. 1

\section{Coupling circuit}

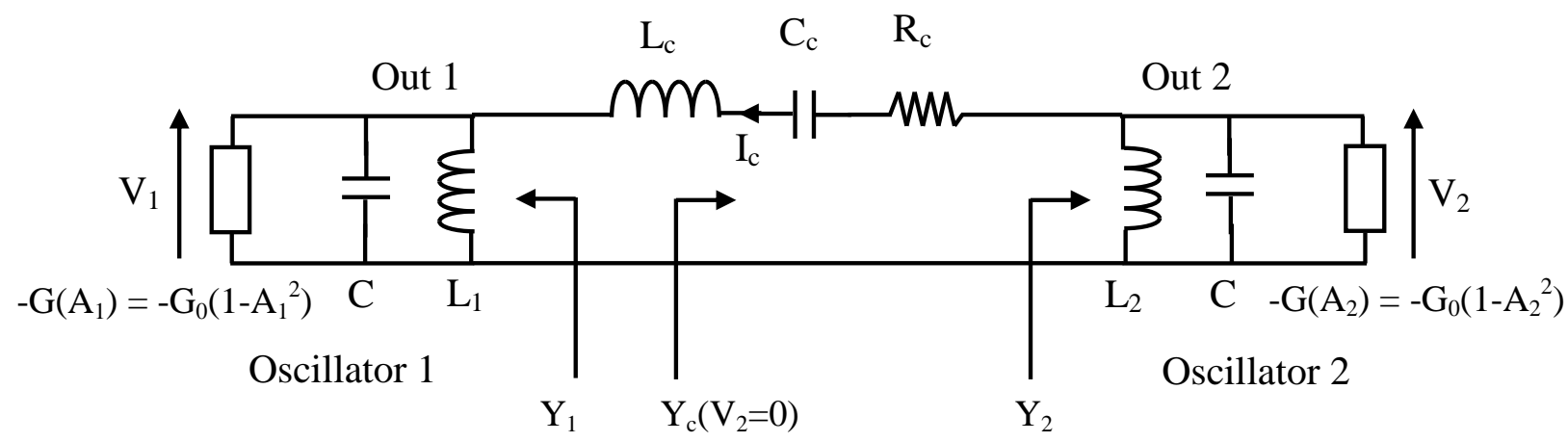

Fig. 2

$\mathrm{i}(\mathrm{t})$

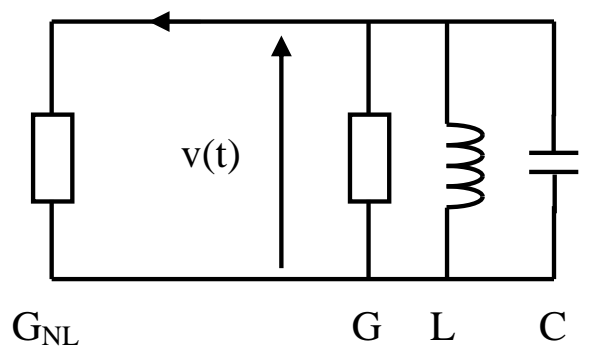


Fig.3

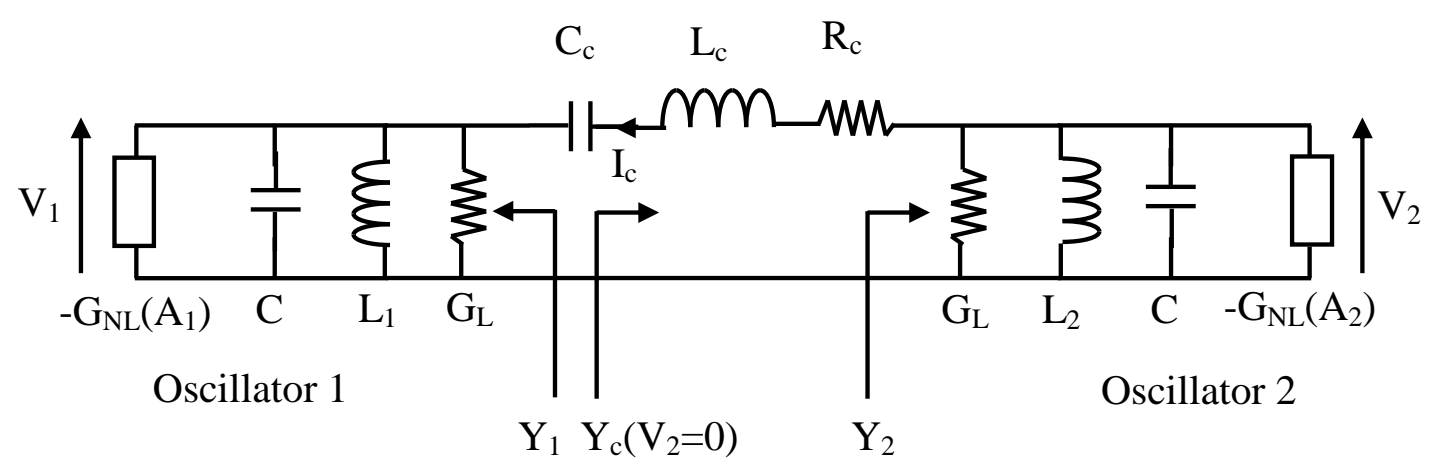

Fig.4

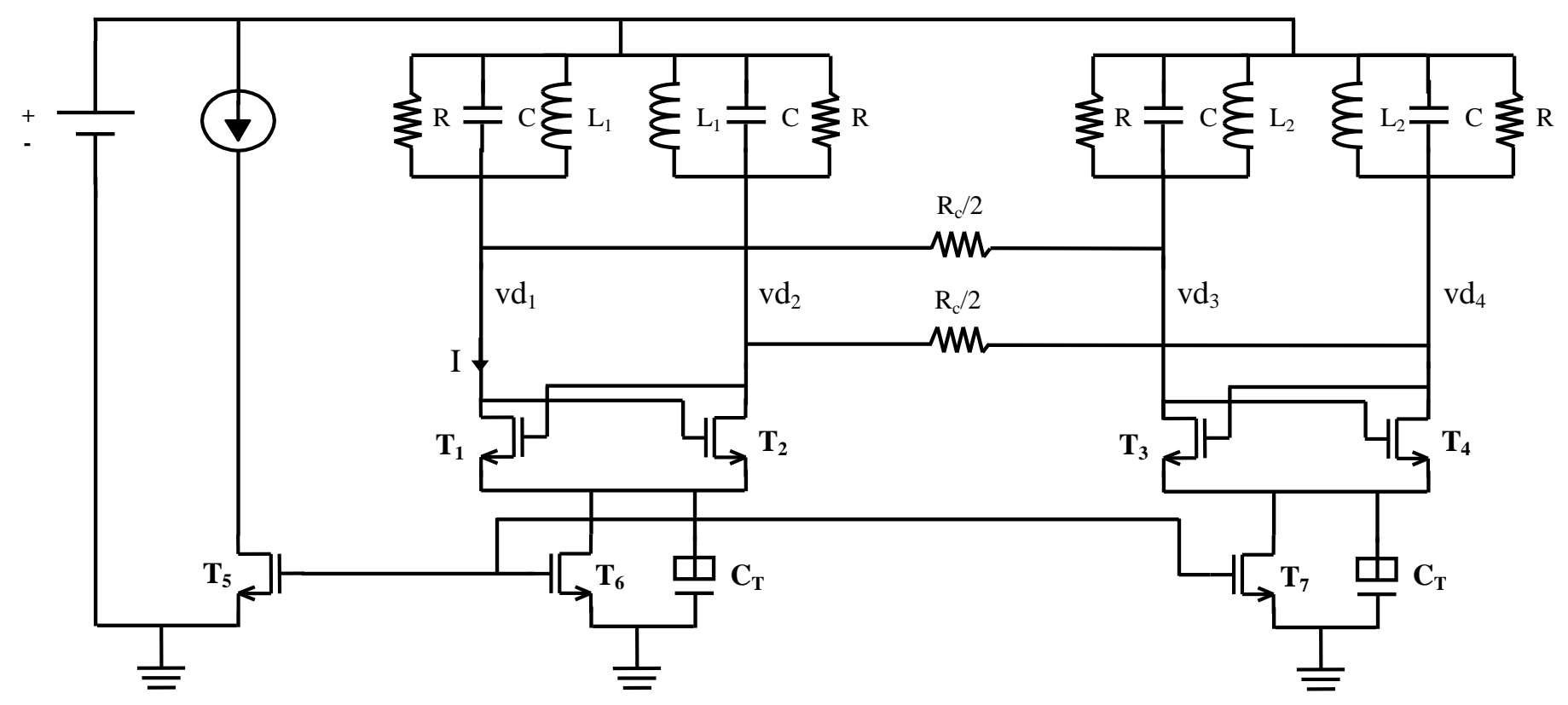

Fig. 5

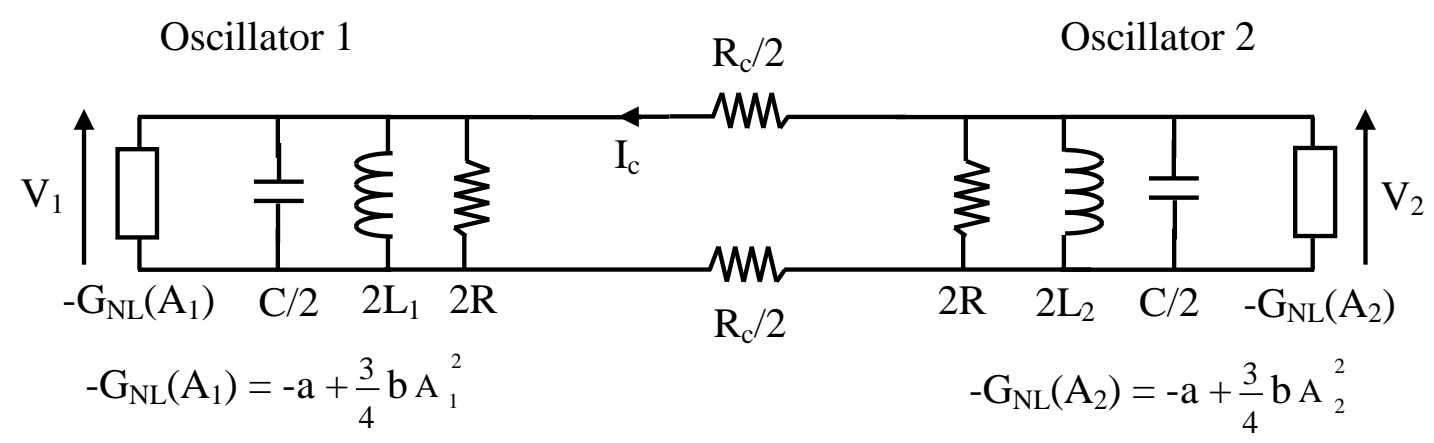


Fig. 6
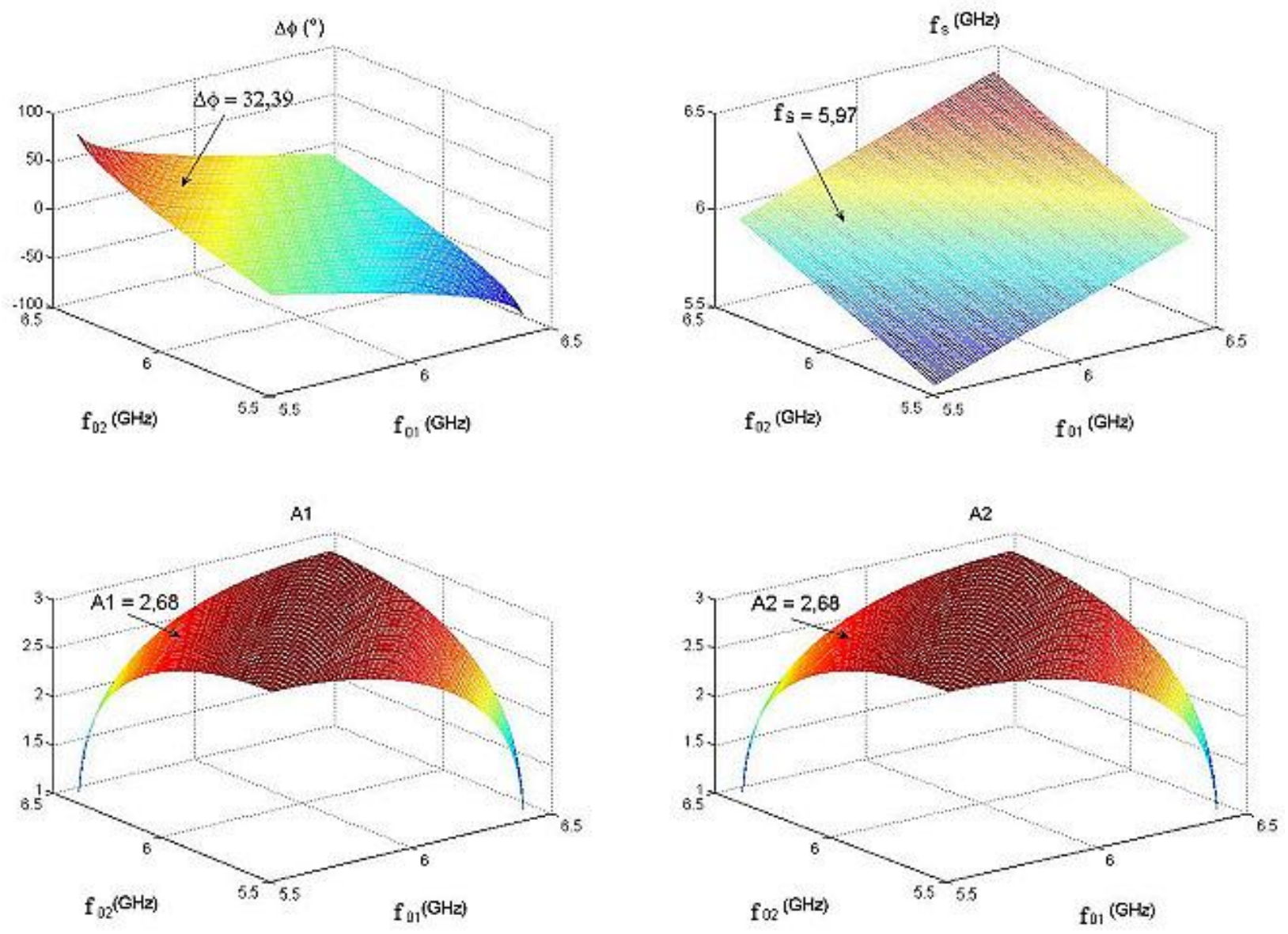
Fig. 7

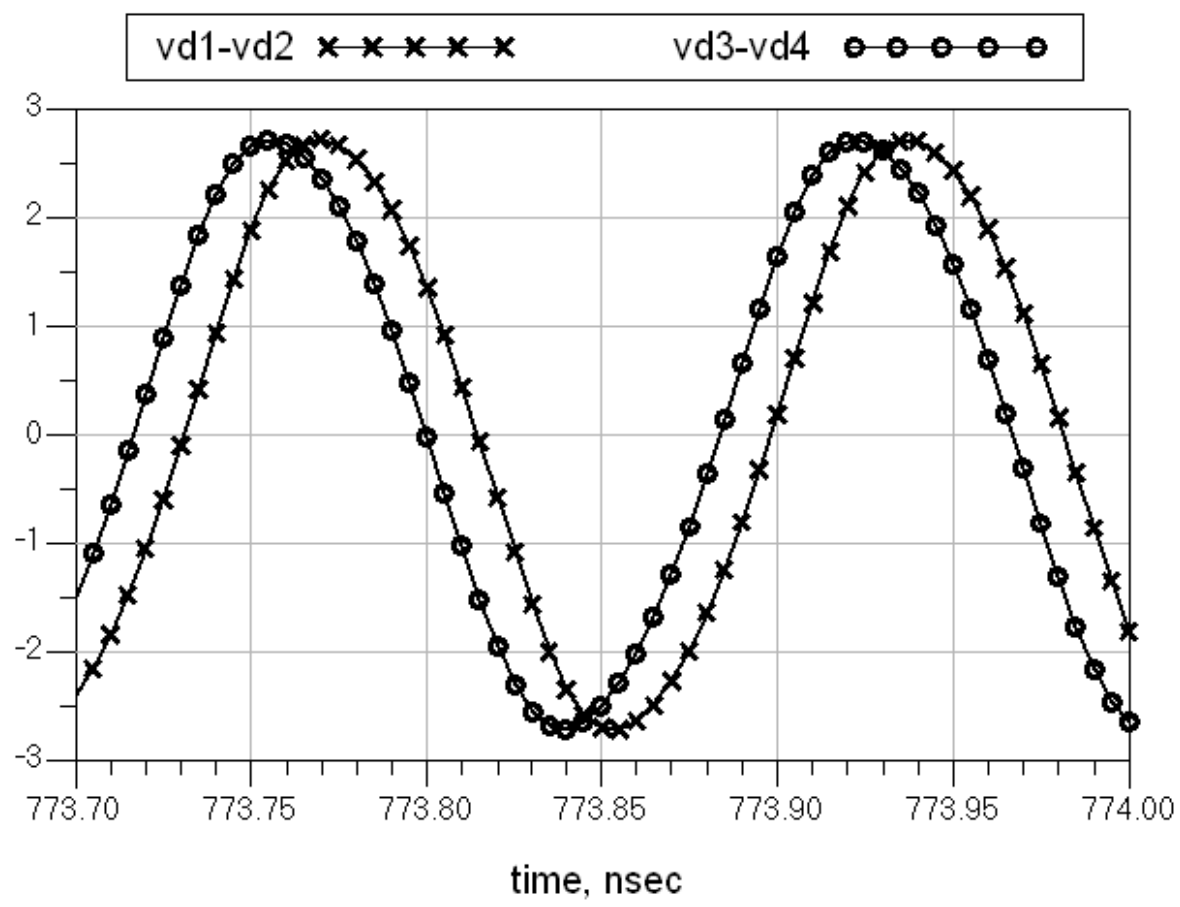

Table I

\begin{tabular}{|c|c|c|c|c|c|c|c|}
\hline \multirow{2}{*}{$\begin{array}{c}\mathbf{f}_{01} \\
(\mathbf{G H z})\end{array}$} & \multirow{2}{*}{$\begin{array}{c}\mathbf{f}_{02} \\
(\mathbf{G H z})\end{array}$} & \multicolumn{3}{|c|}{ ADS } & \multicolumn{3}{|c|}{ CAD tool } \\
\hline & & $\begin{array}{c}\mathbf{f}_{\mathrm{s}} \\
(\mathbf{G H z})\end{array}$ & $\begin{array}{l}\Delta \phi \\
\left(^{\circ}\right)\end{array}$ & $\begin{array}{c}\mathbf{A} \\
(\mathbf{V})\end{array}$ & $\begin{array}{c}\mathbf{f}_{\mathbf{s}} \\
(\mathbf{G H z})\end{array}$ & $\begin{array}{l}\Delta \phi \\
\left({ }^{\circ}\right)\end{array}$ & $\begin{array}{c}\mathbf{A} \\
(\mathbf{V})\end{array}$ \\
\hline 5.97 & 5.97 & 5.96 & $0^{\circ}$ & 2.89 & 5.97 & $0^{\circ}$ & 2.91 \\
\hline 5.91 & 6.03 & 5.96 & $7.18^{\circ}$ & 2.88 & 5.97 & $7.69^{\circ}$ & 2.90 \\
\hline 5.85 & 6.09 & 5.98 & $15.79^{\circ}$ & 2.84 & 5.97 & $15.53^{\circ}$ & 2.86 \\
\hline 5.79 & 6.15 & 5.98 & $24.63^{\circ}$ & 2.78 & 5.97 & $23.69^{\circ}$ & 2.79 \\
\hline 5.73 & 6.21 & 5.98 & $31.23^{\circ}$ & 2.72 & 5.97 & $32.39^{\circ}$ & 2.68 \\
\hline 5.67 & 6.27 & 5.98 & $41.38^{\circ}$ & 2.60 & 5.97 & $42.03^{\circ}$ & 2.52 \\
\hline 5.61 & 6.33 & 5.99 & $53.40^{\circ}$ & 2.43 & 5.97 & $53.46^{\circ}$ & 2.26 \\
\hline
\end{tabular}




\begin{tabular}{|c|c|c|c|c|c|c|c|}
\hline 5.55 & 6.39 & 5.99 & $68.71^{\circ}$ & 2.16 & 5.97 & $69.61^{\circ}$ & 1.75 \\
\hline 5.55 & 6.40 & 5.99 & $69.28^{\circ}$ & 2.15 & 5.97 & $71.53^{\circ}$ & 1.67 \\
\hline 5.54 & 6.41 & 5.99 & $73.90^{\circ}$ & 2.05 & 5.97 & $76.13^{\circ}$ & 1.47 \\
\hline 5.53 & 6.41 & 5.99 & $74.81^{\circ}$ & 2.02 & 5.97 & $79.11^{\circ}$ & 1.32 \\
\hline 5.53 & 6.42 & 5.99 & $81.01^{\circ}$ & 1.85 & 5.97 & $83.29^{\circ}$ & 1.06 \\
\hline
\end{tabular}

Table II

\begin{tabular}{|c|c|c|c|c|c|c|c|}
\hline \multirow{2}{*}{$\begin{array}{c}\mathbf{f}_{01} \\
(\mathbf{G H z})\end{array}$} & \multirow{2}{*}{$\begin{array}{c}\mathbf{f}_{02} \\
(\mathbf{G H z})\end{array}$} & \multicolumn{3}{|c|}{ ADS } & \multicolumn{3}{|c|}{ CAD tool } \\
\hline & & $\begin{array}{c}\mathbf{f}_{\mathrm{s}} \\
(\mathbf{G H z})\end{array}$ & $\begin{array}{l}\Delta \phi \\
\left(^{\circ}\right)\end{array}$ & $\begin{array}{c}\mathbf{A} \\
(\mathbf{V})\end{array}$ & $\begin{array}{c}\mathbf{f}_{\mathrm{s}} \\
(\mathbf{G H z})\end{array}$ & $\begin{array}{l}\Delta \phi \\
\left(^{\circ}\right)\end{array}$ & $\begin{array}{c}\text { A } \\
\text { (V) }\end{array}$ \\
\hline 6.25 & 6.35 & 6.26 & 6.40 & 2.86 & 6.30 & 6.40 & 2.90 \\
\hline 5.56 & 5.80 & 5.65 & 15.53 & 2.87 & 5.68 & 15.53 & 2.86 \\
\hline 5.74 & 6.35 & 6.03 & 41.7 & 2.59 & 6.04 & 42.9 & 2.50 \\
\hline 5.53 & 6.39 & 5.95 & 72.28 & 2.09 & 5.96 & 73.67 & 1.58 \\
\hline
\end{tabular}

